\title{
OPEN Utility of total cell-free DNA levels for surgical damage evaluation in patients with urological surgeries
}

\author{
Sakae Konishi ${ }^{1}$, Takuma Narita ${ }^{1}$, Shingo Hatakeyama ${ }^{2 凶}$, Tohru Yoneyama ${ }^{3}$, \\ Mihoko Sutoh Yoneyama ${ }^{4}$, Yuki Tobisawa ${ }^{1}$, Daisuke Noro ${ }^{5}$, Tendo Sato ${ }^{6}$, Kyo Togashi ${ }^{6}$, \\ Teppei Okamoto ${ }^{1}$, Hayato Yamamoto ${ }^{1}$, Takahiro Yoneyama ${ }^{7}$, Yasuhiro Hashimoto ${ }^{1}$ \& \\ Chikara Ohyama ${ }^{1,2,7}$
}

The evaluation of surgical damage is challenging because of the lack of specific biomarkers. Total cellfree DNA (cfDNA) levels have been reported to increase with external trauma and may be a biomarker for tissue damage. To investigate the utility of perioperative total cfDNA levels in evaluating surgical damage in urological surgeries. This multicenter, prospective, observational study included 196 patients scheduled for urological surgeries between September 2020 and July 2021. The primary outcome was the change in total cfDNA levels before and after urological surgery. The secondary outcome was the effect of surgical type on total cfDNA ratio before and after urological surgery. The postoperative median total cfDNA level of the 196 patients was significantly increased 2.5 -fold compared to the preoperative level $(185.2 \mathrm{ng} / \mathrm{mL}$ vs. $406.7 \mathrm{ng} / \mathrm{mL}, P<0.001)$. The median total cfDNA before/after ratio was greater than four-fold for kidney transplantation, open cystectomy, and open adrenalectomy. The ratio was less than two-fold for laparoscopic adrenalectomy and robot-assisted radical prostatectomy. Major surgery showed a significant postoperative increase in total cfDNA levels, while minor surgery did not. Total cfDNA levels increased 2.5 -fold after urological surgery and it can be used as an acute-phase biomarker for surgical damage.

In recent years, laparoscopic surgery and robot-assisted surgery have become the most common urological procedures ${ }^{1}$. Previous studies have investigated markers for surgical damage, such as operative time, estimated blood loss, length of hospital stay, complications, white blood cell count (WBC), serum C-reactive protein (CRP), cortisol, and cytokines (IL-6, IL-10) $)^{2-4}$. However, these are not definitive markers that reflect the hyperacute invasiveness of surgery. The development of sensitive markers is necessary to compare surgical damage.

Plasma cell-free DNA (cfDNA) has recently been of interest as a liquid biopsy for several cancers. Total cfDNA levels are known to increase not only in cancer ${ }^{5}$ but also in pregnancy ${ }^{6}$, sepsis ${ }^{7}$, organ infarction ${ }^{8}$, and trauma ${ }^{9}$. The main cause of cfDNA increase is cell death ${ }^{10,11}$, which has a half-life of approximately $2 \mathrm{~h}^{12}$. In the field of trauma therapy, post-injury cfDNA levels and the rate of cfDNA reduction are associated with the severity of trauma and the incidence of complications ${ }^{9,13-16}$. Therefore, the total cfDNA level may be a potential marker of tissue damage in the hyperacute phase. Given that elective surgery is a "planned trauma," total cfDNA level may be a potential biomarker for surgical damage assessment. However, only a few studies have examined the changes in cfDNA levels during the perioperative period of surgical procedures ${ }^{17,18}$, and no studies have evaluated the perioperative total cfDNA level in urological surgeries. The present study aimed to examine the utility of perioperative total cfDNA levels for a surgical damage evaluation in urological surgeries.

\footnotetext{
${ }^{1}$ Department of Urology, Hirosaki University Graduate School of Medicine, Hirosaki, Japan. ${ }^{2}$ Department of Advanced Blood Purification Therapy, Hirosaki University Graduate School of Medicine, 5 Zaifu-chou, Hirosaki 036-8562, Japan. ${ }^{3}$ Department of Glycotechnology, Center for Advanced Medical Research, Hirosaki University Graduate School of Medicine, Hirosaki, Japan. ${ }^{4}$ Department of Cancer Immunology and Cell Biology, Oyokyo Kidney Research Institute, Hirosaki, Japan. ${ }^{5}$ Department of Urology, Mutsu General Hospital, Mutsu, Japan. ${ }^{6}$ Department of Urology, Tsugaru General Hospital, Gosyogawara, Japan. ${ }^{7}$ Department of Advanced Transplant and Regenerative Medicine, Hirosaki University Graduate School of Medicine, Hirosaki, Japan. ${ }^{\square}$ email: shingoh@hirosaki-u.ac.jp
} 
A

Minor surgery $n=11$

Adrenalectomy $n=23$

Radical nephrectomy (RN) $n=31$

Hand-assisted Donor nephrectomy

(HALS-DN) $n=9$

Partial nephrectomy (PN) $n=16$

Nephroureterectomy (RNU) $n=6$

Radical cystectomy (RC) $n=17$

Robot-assisted radical

prostatectomy (RARP) $n=73$

Kidney transplantation $(K T) n=10$

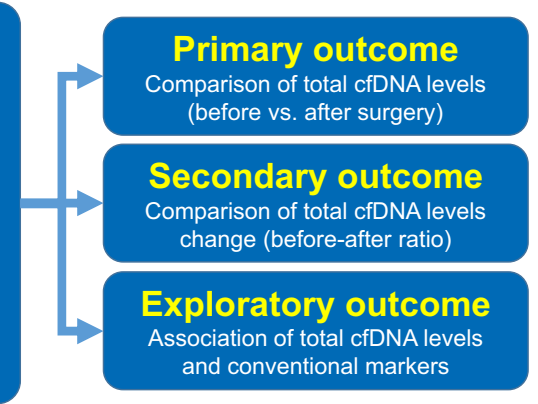

B

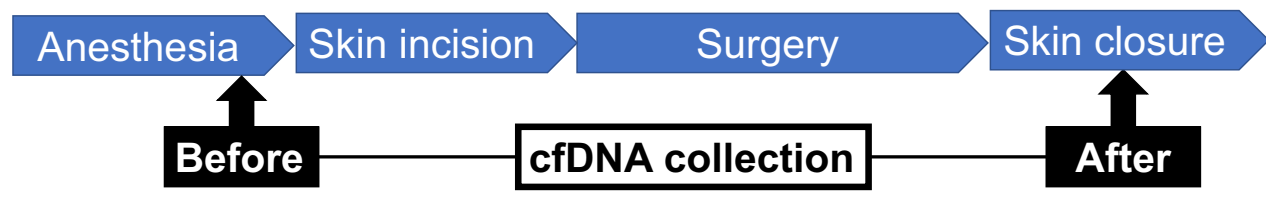

Figure 1. Outline of this study. Outlines of the entire study (A) and blood sampling methods (B).

\begin{abstract}
Results
Stability test of plasma cfDNA at room temperature and $4^{\circ} \mathrm{C}$ storage. At room temperature, the total cfDNA decreased two days after blood collection. On the other hand, the total cfDNA was stable for seven days at $4{ }^{\circ} \mathrm{C}$ (Fig. S1A). The results of electrophoresis are shown in Fig. S1B, S1C. At room temperature storage, the band intensity around $170 \mathrm{bp}$ becomes weaker after the second day. The background level becomes higher, especially for long fragments. When stored at $4{ }^{\circ} \mathrm{C}$, the band intensity around $170 \mathrm{bp}$ and the background level change was limited.
\end{abstract}

Patient demographics and baseline characteristics. Data from 198 Japanese patients who underwent urological surgery at three hospitals were evaluated. Two patients with insufficient cfDNA samples were excluded, and 196 patients were included in the study (Fig. 1).

The median age of patients who underwent urological surgery was 66 years (interquartile range [IQR], 58-72), and $94 \%$ of patients were preoperatively assessed as ECOG-PS 0 . The most common types of surgery were radical prostatectomy $(n=73), R N(n=31)$, adrenalectomy $(n=23)$, and RC $(n=16)$ (Table 1). Patient characteristics and surgical outcomes according to procedure are shown in Table 2.

Primary outcome: the change in total cfDNA level before and after urological surgery. As a preliminary study, the trends of total cfDNA levels in major surgeries (robot-assisted radical cystectomy (RARC), RARP, and laparoscopic RN) within four days were evaluated. Results showed that the total cfDNA level increased immediately after surgery and promptly decreased the next day (Fig. S2A). In all eligible patients, the postoperative median total cfDNA levels were significantly increased (Fig. 2, $185.2 \mathrm{ng} / \mathrm{mL}$ vs. $406.7 \mathrm{ng} / \mathrm{mL}$, $P<0.001$ ), which was 2.5 -fold higher than the preoperative level. There was no significant increase in postoperative median total cfDNA levels in patients who underwent minor surgeries (Fig. 2, $214.1 \mathrm{ng} / \mathrm{mL}$ vs. $297.2 \mathrm{ng} / \mathrm{mL}$, $P=0.560$ ); however, there was a significant increase in total cfDNA levels in most major surgeries. A significant postoperative rise in total cfDNA levels was found in laparoscopic adrenalectomy (Fig. S2B, $P<0.001$ ), open RN (Fig. S2C, $P=0.008$ ), laparoscopic RN (Fig. S2C, $P<0.001$ ), robot-assisted PN (Fig. S2D, $P<0.001$ ), robotassisted RC (Fig. S2E, $P<0.001$ ), RARP (Fig. S2F, $P<0.001$ ), HALS-DN (Fig. S2G, $P<0.001$ ), and KT (Fig. S2H, $P=0.004)$. No significant difference in total cfDNA levels was observed in patients with RNU (Fig. S2I, $P=0.18$ ). Statistical difference was not evaluated in open adrenalectomy (Fig. S2B), open PN (Fig. S2D), and open RC (Fig. S2E) because of the small number of patients $(n<5)$.

Secondary outcomes: The difference in total cfDNA before/after ratio depending on the type of surgery (open, laparoscopic, or robot-assisted). Figure 3A summarizes the ratios of postoperative total cfDNA levels compared to preoperative levels according to the type of surgery. The median cfDNA before/ after ratio was greater than four-fold for KT, open cystectomy, and open adrenalectomy. The ratio was less than two-fold for laparoscopic adrenalectomy and RARP.

The difference in total cfDNA before/after ratio in the same organ according to the type of surgery was then investigated. There was no significant difference in the total cfDNA before/after ratio between open and laparoscopic/robot-assisted surgery of the kidney (Fig. 3B, 2.6-fold vs. 3.0-fold, $P=0.88$ ). For pelvic surgery of the prostate and bladder, the total cfDNA before/after ratio of open RC tended to be higher than that of RARP and RARC (Fig. 3C). When RARC and RARP were compared, RARC showed a significantly higher ratio (Fig. 3C, 3.4-fold vs. 2.0 -fold, $P=0.046$ ). For adrenal surgery, there was a trend towards higher ratios for open surgery 


\begin{tabular}{|c|c|}
\hline n & 196 \\
\hline Age, years (IQR) & $66(58-72)$ \\
\hline Male, $\mathrm{n}$ & $153(78 \%)$ \\
\hline \multicolumn{2}{|l|}{ ECOG-PS } \\
\hline 0 & $183(94 \%)$ \\
\hline 1 & $11(5 \%)$ \\
\hline 2 & $2(1 \%)$ \\
\hline 3 & 0 \\
\hline 4 & 0 \\
\hline Major surgery, n & $185(94 \%)$ \\
\hline Minor surgery, $\mathrm{n}$ & $11(6 \%)$ \\
\hline \multicolumn{2}{|l|}{ Type of surgery, $n$} \\
\hline Radical Prostatectomy & $73(37)$ \\
\hline \multicolumn{2}{|l|}{ Nephrectomy } \\
\hline Radical & $31(16)$ \\
\hline Partial & $16(8)$ \\
\hline Donor & $9(5)$ \\
\hline Adrenalectomy & $23(12)$ \\
\hline Radical Cystectomy & $17(8)$ \\
\hline Kidney transplantation & $10(5)$ \\
\hline Radical Nephroureterectomy & $6(3)$ \\
\hline TURBT & $6(3)$ \\
\hline High orchiectomy & $4(2)$ \\
\hline Other & $1(1)$ \\
\hline
\end{tabular}

Table 1. Background of patients. $I Q R$ interquartile range, ECOG-PS Eastern cooperative oncology group performance status, TURBT transurethral resection of bladder tumor.

\begin{tabular}{|c|c|c|c|c|c|c|c|c|c|c|}
\hline & \multirow[b]{2}{*}{ n } & \multicolumn{4}{|c|}{ TNM classification* } & \multicolumn{5}{|c|}{ Postop. complications } \\
\hline & & T1 & T2 & T3,4 & N1 & M1 & Surgical time, $\min (\mathrm{IQR})$ & Blood loss, g (IQR) & Any grade & Grade $\geq 3$ \\
\hline Minor surgery & 11 & 6 & 2 & 2 & 1 & 0 & $47(23-65)$ & $5(5-5)$ & 0 & 0 \\
\hline RARP & 73 & 27 & 12 & 34 & 0 & 4 & $183(163-201)$ & $30(10-50)$ & 11 & 0 \\
\hline Open radical cystectomy & 4 & 1 & 1 & 2 & 0 & 0 & 170 & 2088 & 0 & 0 \\
\hline RARC & 13 & 1 & 4 & 8 & 2 & 0 & $357(316-404)$ & $630(325-790)$ & 6 & 1 \\
\hline Open RN & 8 & 3 & 0 & 5 & 0 & 3 & $147(131-170)$ & $145(28-332)$ & 2 & 0 \\
\hline Laparoscopic RN & 23 & 18 & 1 & 4 & 0 & 2 & $184(167-203)$ & $20(5-40)$ & 2 & 0 \\
\hline HALS-DN & 9 & & & & & & $217(23-65)$ & $12(23-65)$ & 0 & 0 \\
\hline Open adrenalectomy & 4 & & & & & & $179(100-370)$ & $660(100-1715)$ & 0 & 0 \\
\hline Laparoscopic adrenalectomy & 19 & & & & & & $137(113-154)$ & $5(5-10)$ & 2 & 2 \\
\hline RAPN & 13 & 13 & 0 & 0 & 0 & 0 & $170(140-201)$ & $15(10-50)$ & 0 & 0 \\
\hline Open partial nephrectomy & 3 & 1 & 0 & 0 & 0 & 0 & 155 & 390 & & \\
\hline Kidney transplantation & 10 & & & & & & $308(276-370)$ & $190(125-500)$ & 1 & 0 \\
\hline Laparoscopic RNU & 6 & 0 & 2 & 4 & 0 & 0 & 209 (194-255) & $50(5-73)$ & 1 & 0 \\
\hline
\end{tabular}

Table 2. Patient characteristics and surgical outcomes by type of surgeries. $I Q R$ interquartile range, $R A R P$ Robot-assisted radical prostatectomy, $R A R C$ Robot-assisted radical cystectomy, $R N$ Radical nephrectomy, $H A L S-D N$ Hand-assisted laparoscopic donor nephrectomy, RAPN Robot-assisted partial nephrectomy, RNU Radical nephroureterectomy. ${ }^{*} \mathrm{TNM}$ classification for patients with non-malignant tumors was not performed.

(Fig. 3D). The median total cfDNA before and after surgery for other surgeries was 1.0-fold for minor surgery, 2.3-fold for laparoscopic RNU, and 4.7-fold for KT (Fig. 3E).

Exploratory outcome. There was no significant correlation between the postoperative increase ratio of WBC (Fig. 4A), increase ratio of CRP (Fig. 4B), surgical time (Fig. 4C), and estimated blood loss (Fig. 4D) with total cfDNA before/after ratio, regardless of the type of surgery. There was also no correlation between the conventional surgical damage markers and postoperative total cfDNA levels. 


\section{Change in total cfDNA level}

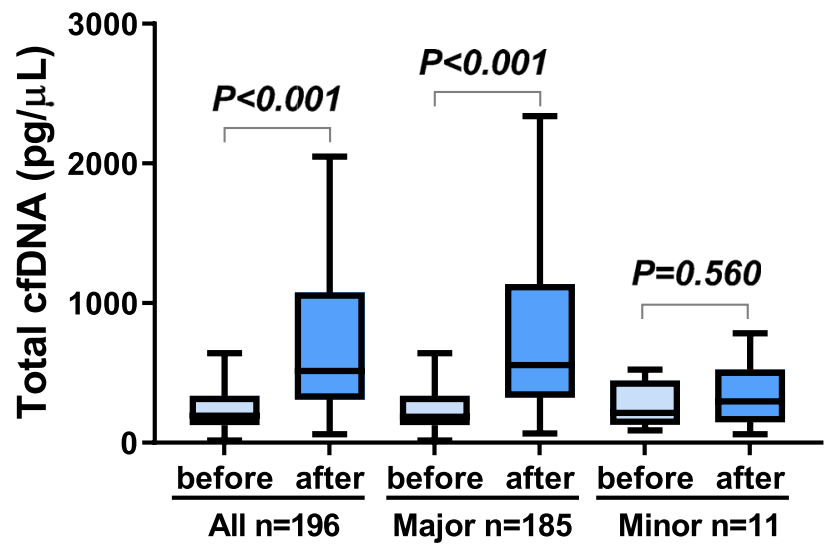

Figure 2. The difference of total cell-free DNA levels before and after surgery. Difference in total cell-free DNA (cfDNA) levels before and after urologic surgery.

\section{A Summary of total cfDNA ratios}

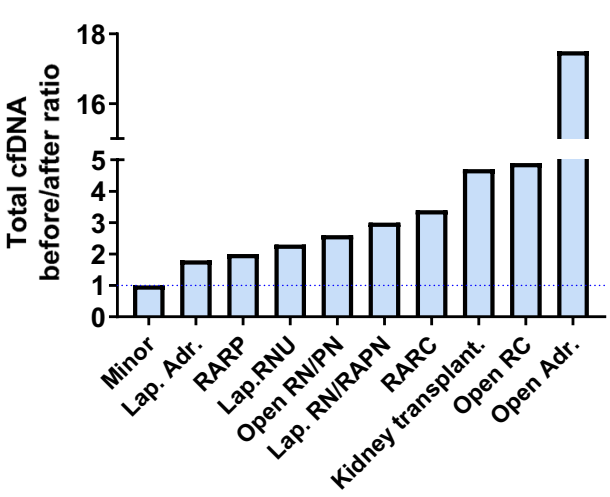

B Open vs. Lap./Robotic (RN/PN)

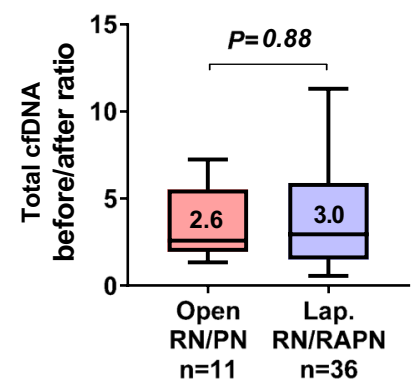

D Open vs. Lap. (Adrenal)

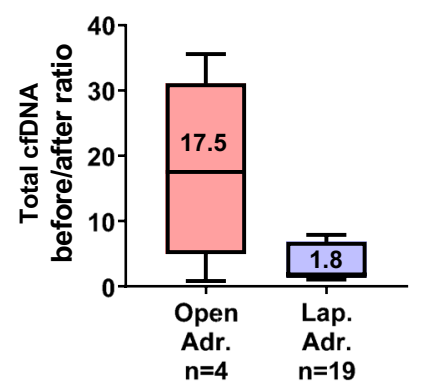

C Open vs. Lap./Robotic (Pelvis)

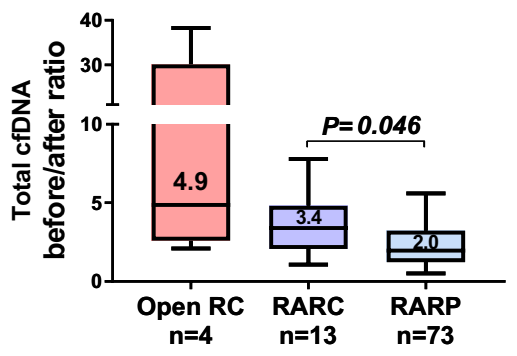

E

Other surgery

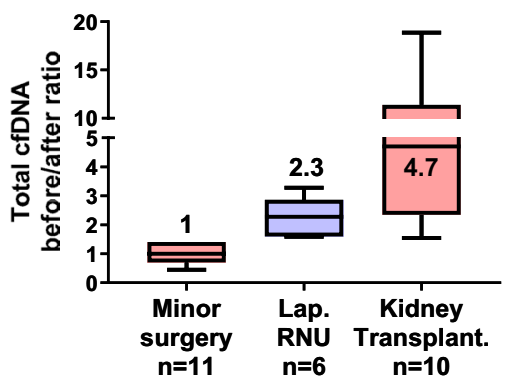

Figure 3. Comparison of total cfDNA ratios before and after surgery for each surgery types. Summary of total cell-free DNA (cfDNA) ratios before and after surgery according to type of surgery (A). Comparisons of total cfDNA before/after ratios for open and laparoscopic/robotic kidney surgery $(\mathbf{B})$, open and robot-assisted radical cystectomy and robot-assisted radical prostatectomy $(\mathbf{C})$, open and laparoscopic adrenalectomy (D), and other surgeries (minor surgery, laparoscopic radical nephroureterectomy, kidney transplantation) (E).

Total cfDNA before/after ratios were not significantly associated with or without postoperative complications for RARP (Fig. S3A) and RARC (Fig. S3B).

\section{Discussion}

The current study is the first to investigate the utility of perioperative total cfDNA levels for the evaluation of surgical damage in urological surgeries. Previous study have used to assess the extent of damage in the trauma field ${ }^{9,13-16}$. A review article examining the utility of post-traumatic cfDNA measurement in 904 patients from 14 observational studies found a correlation between total cfDNA level and severity of injury, post-traumatic complications, mortality, and length of stay in the intensive care unit ${ }^{9}$. However, a strong limitation of these 


\section{A Association of total cfDNA ratio with WBC}



\section{Association of total cfDNA ratio with surgical time}

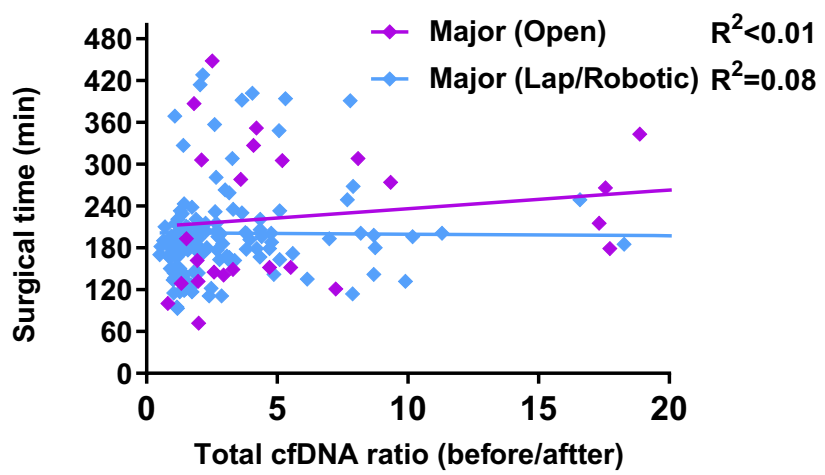

B Association of total cfDNA ratio with CRP



\section{Association of total cfDNA ratio with blood loss}

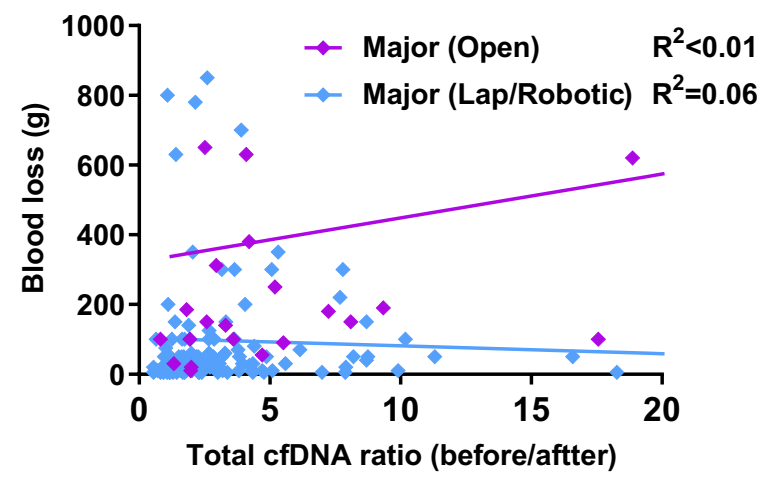

Figure 4. Pearson correlation plots of cell-free DNA ratio before and after surgery with conventional markers. A linear relationship between the cell-free DNA increasing ratio and conventional markers by scatterplot and linear regression analysis with correlation coefficient R. Markers examined were WBC ratio (before and after surgery) (A), CRP ratio (before and after surgery) (B), surgical time (C) and blood loss (D).

trauma studies is the lack of pre-traumatic cfDNA levels. The review article also commented that cfDNA should be examined pre- and post-surgery to assess the extent of damage ${ }^{9}$. Accordingly, the current study evaluated the pre- and postoperative values of total cfDNA for elective surgeries. Results showed that the postoperative total cfDNA level was significantly higher than the preoperative level (Fig. 1). The median total cfDNA before/ after ratio tended to be higher in open surgeries and was almost the same value in minor surgeries. As cfDNA is presumed to be released by cell injury, necrosis, or inflammation ${ }^{10,11}$, total cfDNA levels might be helpful in evaluating surgical damage.

The utility of total cfDNA levels for the assessment of surgical damage needs further discussion. Only one previous article on the changes in total cfDNA levels pre- and post-surgery is present. A prospective exploratory study of 10 patients undergoing arthroscopic surgery (orthopedic knee replacement surgery, $n=5$; orthopedic hip replacement surgery, $n=5$ ) reported that the median total cfDNA levels increased immediately after surgery and decreased on the first postoperative day ${ }^{17}$. These results were similar to those of the current study, and total cfDNA levels might be useful to assess external damage as an acute-phase biomarker. However, there is the lack of available information regarding the source of cfDNA, which is speculated to be released from damaged cells and immune cells. Therefore, it is difficult to conclude that the total cfDNA level is a useful biomarker for surgical damage. Further studies are necessary to address its role in the tissue damage.

Results showed a difference in total cfDNA levels between open and laparoscopic/robotic surgeries (Fig. 3A). The total cfDNA increase ratio was minimal for minor surgery, moderate for laparoscopic/robot-assisted surgery, and maximal for open surgery. This trend may represent surgical damage, reflecting the number of cell deaths. First, a small skin incision might be a key factor for surgical damage, and open surgery may have a higher total cfDNA level. However, nephrectomy showed no significant difference in the total cfDNA before/after ratio between the open and laparoscopic/robotic surgeries (Fig. 3B). This result may suggest similar tissue damage in kidney surgery regardless of the procedure. The similar perirenal peeling area might be the reason, but little is known about the effect of skin incision or peeling on total cfDNA levels. Second, small cases of open RN/PN under different incisions (abdominal midline incision or oblique lumbar incision) are limitations of this study. Further studies are required to answer these questions. 
The researchers speculated that surgical damage can be associated with conventional parameters such as postoperative WBC count, CRP level, surgical time, and blood loss. However, no relationship was found between total cfDNA before/after ratio in major surgery and these parameters (Fig. 4). These findings suggest that total cfDNA levels may have different properties than inflammatory markers, such as WBC and CRP. As the total cfDNA rises and falls rapidly, it might be associated with cortisol and cytokines (IL-6 and IL-10). Several studies have suggested a potential relationship between surgical stress and cortisol/cytokines ${ }^{2-4}$. Future investigations should address the potential role of cortisol, IL-6, and total cfDNA levels in surgical damage.

The association between total cfDNA level and postoperative complications is the object of interest. As a previous study showed a positive association between the total cfDNA level and post-traumatic complications ${ }^{13,19}$, elevated postoperative total cfDNA levels may be related to a higher rate of postoperative complications. However, results showed that the total cfDNA before/after ratio was not significantly different between patients with and without postoperative complications (Fig. S3). As the total cfDNA level falls rapidly after surgery, continuous measurements might be more useful than single point analysis to predict postoperative events. The small number of events ( $n=3$ in grade $\geq 3$ ) was the major limitation of this study, and further study is needed to investigate the utility of total cfDNA level on predicting postoperative complications.

This study has several limitations. First, a small number of cases could be a source of bias. In particular, the number of patients undergoing open surgery was significantly lower owing to the recent trend of minimally invasive surgery. However, this is the first study to investigate perioperative total cfDNA levels and its usefulness in urological surgeries. Further large-scale validation studies and investigation of the relationship between cfDNA levels and surgical outcomes are needed.

In conclusion, total cfDNA levels increased 2.5-fold after urological surgery. The cfDNA increase ratio was minimal for minor surgery, moderate for laparoscopic/robot-assisted surgery, and maximal for open surgery. Total cfDNA levels may be a new acute-phase biomarker for surgical damage. Further studies are required.

\section{Methods}

We performed a multicenter, prospective, observational study in accordance with the ethical standards outlined in the Declaration of Helsinki. The ethics committee of the Hirosaki University School of Medicine approved this study at Hirosaki University School of Medicine, Mutsu general hospital and Tsugaru general hospital (authorization number: 2020-258). Written consents were obtained from all participants.

Study population and patient selection. An outline of this study is shown in Fig. 1A. Patients scheduled for urological surgeries at one academic and two satellite centers between September 2020 and July 2021 were evaluated. The urological surgeries included in this study were transurethral resection of the bladder tumor (TURBT), high orchiectomy, adrenalectomy, radical nephrectomy (RN), hand-assisted laparoscopic donor nephrectomy (HALS-DN), partial nephrectomy (PN), radical nephroureterectomy (RNU), radical cystectomy (RC), robot-assisted radical prostatectomy (RARP), and kidney transplantation (KT). TURBT and high orchiectomy were defined as minor surgeries, while the rest were defined as major surgeries. Patients with insufficient cfDNA were excluded. The selection of open, laparoscopic, or robot-assisted surgery was discussed at the meeting in the researchers' department prior to surgery.

Variable evaluations. The following parameters of patients were analyzed: age, sex, Eastern Cooperative Oncology Group performance status (ECOG-PS), history of neoadjuvant therapy, operation time, intraoperative estimated blood loss, postoperative peripheral blood data, serum albumin, and CRP. Tumor stage was assigned based on the 2009 TNM classification of the Union for International Cancer Control. Postoperative complications were evaluated using the Clavien-Dindo classification.

Blood Sampling and Plasma preparation. Blood (7 cc) was collected from the radial arterial line using a BD Vacutainer ${ }^{\infty}$ Barricor $^{\text {Tix }}$ plasma blood collection tube with heparin lithium prior to surgery (before skin incision) and at the end of surgery (at skin closure) (Fig. 1B). In some patients $(n=10)$, peripheral venous blood was collected on postoperative days one and four to confirm the trend of total cfDNA levels. The blood was centrifuged at $3000 \mathrm{~g}$ for $10 \mathrm{~min}$ immediately after blood collection, and total cfDNA levels were either measured immediately or the centrifuged blood was stored at $4{ }^{\circ} \mathrm{C}$.

Stability test of plasma cfDNA at room temperature and $4^{\circ} \mathrm{C}$ storage. We investigated the stability of the total cfDNA. Each of the three plasma samples was divided into six aliquots and stored at room temperature and $4{ }^{\circ} \mathrm{C}$. We performed electrophoresis of each blood sample on day 0 (immediately), 1, 2, 3, 6 and, 7 to examine the changes in cfDNA. Also, the electropherogram at day 0,3 , and 7 were evaluated.

Plasma cfDNA extraction and cfDNA characteristics. The AB MagMAX Cell-Free DNA Isolation Kit (Applied Biosystems, Foster City, CA, USA) was used to extract cfDNA from the plasma sample (1 mL). The total cfDNA levels were analyzed using an Agilent High Sensitivity DNA Kit and Agilent Bioanalyzer 2100 (Agilent Technologies Japan, Ltd., Tokyo, Japan). The detection limit of cfDNA in this experimental system is $5 \mathrm{pg} / \mu \mathrm{L}$, and the fragment length can be measured from 50 to $7000 \mathrm{bp}$. We measured a fragment of the mononucleosomes around $170 \mathrm{bp}$. The range of fragment length and the calculation of cfDNA concentrations were determined automatically by 2100 Expert Software ver. B.02.08.SI648(SR2) (Aglient Technologies, Santa Clara, United States). Two experimenters in a single institution (Hirosaki University) analyzed the total cfDNA levels based on the same protocol. 
Outcome measurements. The primary outcome was the change in total cfDNA levels before and after urological surgery, and the secondary outcome was the difference in total cfDNA before/after ratio, depending on the type of surgery. The exploratory outcomes were the association of total cfDNA before/after ratio and conventional markers before operation/after operation ratio (white blood cell counts, CRP, operation time, and estimated blood loss) and the association of total cfDNA before/after ratio and postoperative complications. White blood cell counts and CRP data were taken $1 \mathrm{~h}$ and $12-18 \mathrm{~h}$ after the surgery, respectively.

Statistical analysis. Statistical analyses of clinical data were performed using BellCurve for Excel (Social Survey Research Information Co., Ltd., Tokyo, Japan) and GraphPad Prism v. 9.12 (GraphPad Software, San Diego, CA, USA). Categorical variables were reported as percentages and compared using Fisher's exact test. Quantitative data were expressed as medians with quartiles. Differences between the groups were statistically compared using the Mann-Whitney $U$ test. Statistical significance was set at $P<0.05$. A linear relationship between the two variables was evaluated by scatterplot and linear regression analysis with correlation coefficient $\mathrm{R}$. Absolute R values of $0.00-0.24,0.25-0.49,0.50-0.74$, and $0.75-1.00$ were defined as none to very weak, weak, moderate, and strong linear relationships, respectively.

Consent for Publication. All authors approved for the publication.

\section{Data availability}

The minimal data set of the present study is available on request.

Received: 12 September 2021; Accepted: 28 October 2021

Published online: 11 November 2021

\section{References}

1. Rassweiler, J. J. \& Teber, D. Advances in laparoscopic surgery in urology. Nat. Rev. Urol. 13, 387-399 (2016).

2. Miyake, H. et al. Comparison of surgical stress between laparoscopy and open surgery in the field of urology by measurement of humoral mediators. Int. J. Urol. 9, 329-333 (2002).

3. Porcaro, A. B. et al. Robotic-assisted radical prostatectomy is less stressful than the open approach: results of a contemporary prospective study evaluating pathophysiology of cortisol stress-related kinetics in prostate cancer surgery. J. Robot. Surg. 9, 249-255 (2015).

4. Rubinstein, M. et al. Prospective, randomized comparison of transperitoneal versus retroperitoneal laparoscopic adrenalectomy. J. Urol. 174, 442-5; discussion 445 (2005).

5. Corcoran, R. B. \& Chabner, B. A. Application of cell-free DNA analysis to cancer treatment. N. Engl. J. Med. 379, 1754-1765 (2018).

6. Bianchi, D. W. \& Chiu, R. W. K. Sequencing of circulating cell-free DNA during pregnancy. N. Engl. J. Med. 379, 464-473 (2018).

7. Dwivedi, D. J. et al. Prognostic utility and characterization of cell-free DNA in patients with severe sepsis. Crit. Care 16, R151 (2012).

8. Chang, C.P.-Y. et al. Elevated cell-free serum DNA detected in patients with myocardial infarction. Clin. Chim. Acta 327, 95-101 (2003).

9. Gögenur, M., Burcharth, J. \& Gögenur, I. The role of total cell-free DNA in predicting outcomes among trauma patients in the intensive care unit: a systematic review. Crit. Care 21, 14 (2017)

10. Fournié, G. J. et al. Plasma DNA as a marker of cancerous cell death. Investigations in patients suffering from lung cancer and in nude mice bearing human tumours. Cancer Lett. 91, 221-227 (1995).

11. Fournié, G. J., Martres, F., Pourrat, J. P., Alary, C. \& Rumeau, M. Plasma DNA as cell death marker in elderly patients. Gerontology 39, 215-221 (1993).

12. Diehl, F. et al. Circulating mutant DNA to assess tumor dynamics. Nat. Med. 14, 985-990 (2008).

13. Lo, Y. M., Rainer, T. H., Chan, L. Y., Hjelm, N. M. \& Cocks, R. A. Plasma DNA as a prognostic marker in trauma patients. Clin. Chem. 46, 319-323 (2000).

14. Lam, N. Y. L., Rainer, T. H., Chan, L. Y. S., Joynt, G. M. \& Lo, Y. M. D. Time course of early and late changes in plasma DNA in trauma patients. Clin. Chem. 49, 1286-1291 (2003).

15. Macher, H. et al. Role of early cell-free DNA levels decrease as a predictive marker of fatal outcome after severe traumatic brain injury. Clin. Chim. Acta 414, 12-17 (2012).

16. Ren, B. et al. Is plasma cell-free DNA really a useful marker for diagnosis and treatment of trauma patients?. Clin. Chim. Acta 424, 109-113 (2013).

17. Brodbeck, K. et al. Quantitative analysis of individual cell-free DNA concentration before and after penetrating trauma. Int. J. Legal Med. 133, 385-393 (2019).

18. Henriksen, T. V. et al. The effect of surgical trauma on circulating free DNA levels in cancer patients-implications for studies of circulating tumor DNA. Mol. Oncol. 14, 1670-1679 (2020).

19. Rainer, T. H. Plasma DNA, prediction and post-traumatic complications. Clin. Chim. Acta 313, 81-85 (2001).

\section{Acknowledgements}

The authors would like to thank Satomi Sakamoto, Yukie Nishizawa and Yuki Fujita for their invaluable help with data and sample collection. We would like to thank Editage (www.editage.com) for English language editing.

\section{Author contributions}

All authors reviewed the manuscript. S.K.: manuscript writing, data collection T.N.: manuscript writing, data analysis, data collection. S.H.: Project development, manuscript writing, data analysis, data collection. T.Y.: Project development, Data collection. M.S.Y.: Data collection. Y.T.: Data collection. D.N.: Data collection. T.S.: Data collection. K.T.: Data collection. T.O.: Data collection. H.Y.: Data collection. T.Y.: Data collection. Y.H.: Data collection. C.O.: Data collection, critical review. 


\section{Funding}

Grants-in-Aid for Scientific Research 20K18130 (T.N.), 21 K09339 (T.Y.) and 20K09517 (S.H.) from the Japan Society for the Promotion of Science.

\section{Competing interests}

The authors declare no competing interests.

\section{Additional information}

Supplementary Information The online version contains supplementary material available at https://doi.org/ 10.1038/s41598-021-01430-z.

Correspondence and requests for materials should be addressed to S.H.

Reprints and permissions information is available at www.nature.com/reprints.

Publisher's note Springer Nature remains neutral with regard to jurisdictional claims in published maps and institutional affiliations.

(c) (i) Open Access This article is licensed under a Creative Commons Attribution 4.0 International License, which permits use, sharing, adaptation, distribution and reproduction in any medium or format, as long as you give appropriate credit to the original author(s) and the source, provide a link to the Creative Commons licence, and indicate if changes were made. The images or other third party material in this article are included in the article's Creative Commons licence, unless indicated otherwise in a credit line to the material. If material is not included in the article's Creative Commons licence and your intended use is not permitted by statutory regulation or exceeds the permitted use, you will need to obtain permission directly from the copyright holder. To view a copy of this licence, visit http://creativecommons.org/licenses/by/4.0/.

(C) The Author(s) 2021 